\title{
Spoilers inteligentes e sustentáveis para expansão urbana no interior da América do Sul: Resenha para Várzea Grande, MTI
}

\section{Intelligent and sustainable spoilers for urban expansion in the interior of South America: Review for Várzea Grande, MT}

\author{
Machado, Fernando'; Carvalho, Alessandra2; Barden, Daniela ${ }^{3}$ \\ ' UNIVAG - Centro Universitário de Várzea Grande; Várzea Grande-MT, Brasil; \\ fernando.machado@univag.edu.br \\ 2 UNIVAG - Centro Universitário de Várzea Grande; \\ alessandra.inovi@gmail.com \\ 3 UNIVAG - Centro Universitário de Várzea Grande; \\ daniela.barden@univag.edu.br
}

\begin{abstract}
RESUMO
O artigo sobre morfologia urbana no Estado de Mato Grosso tem como objetivo refletir a expansão urbana e memória local do conjunto de saberes e práticas sob o ponto de vista de cidades inteligentes e sustentáveis. É uma análise teórica do processo de urbanização da cidade de Várzea Grande, Mato Grosso. A expansão urbana na área central da América do Sul é igual à urbanização mundial com características semelhantes, mas os agentes são diferentes, indicando o regionalismo como fator determinante de novas ações sob os aspectos das cidades inteligentes e sustentáveis. Dessa maneira, o estudo apresentado contribuirá na elaboração e na adoção de cenários de planejamento urbano para cidades do porte de Várzea Grande, possibilitando corrigir erros e apontar novos caminhos para o crescimento das cidades.
\end{abstract}

Palavras-chave: Expansão Urbana, Cidade Inteligente, Cidade Sustentável.

\begin{abstract}
The article on urban morphology in the State of Mato Grosso aims to reflect the urban expansion and local memory of a set of knowledge and practices from the point of view of intelligent and sustainable cities. It is a theoretical analysis of the urbanization process of the city of Várzea Grande, Mato Grosso. The urban expansion in the central area of South America is equal to world urbanization with similar characteristics, but the agents are different, indicating regionalism as a determining factor of new actions under the aspects of the intelligent and sustainable cities. In this way, the study presented will contribute to the elaboration and adoption of urban planning scenarios for towns of the size of Várzea Grande, making it possible to correct errors and point out new paths for city growth.
\end{abstract}

\footnotetext{
${ }^{1}$ MACHADO, Fernando 1; CARVALHO, Alessandra 2; BARDEN, Daniela 3. Spoilers inteligentes e sustentáveis para expansão urbana no interior da América do Sul: Resenha para Várzea Grande, MT. In: II SIMPÓSIO NACIONAL DE GESTÃO E ENGENHARIA URBANA: SINGEURB, 2019, São Paulo. Anais... Porto Alegre: ANTAC, 2019.
} 
Keywords: Urban Expansion, Smart City, Sustainable City.

\section{INTRODUÇÃO}

O processo de urbanização intensificou-se e acelerou-se em todo o mundo nas últimas décadas, especialmente nos países subdesenvolvidos, conforme Ribeiro et al. (2018). Assim, a cidade, ao se tornar a maior expressão das modificações realizadas pelo ser humano, reflete a conturbada relação entre o homem e a natureza.

No Brasil, o processo de urbanização não ocorre somente nos eixos Rio-São Paulo. No final do século XX é possível identificar a aceleração da urbanização no interior do Brasil. A cidade de Várzea Grande, Mato Grosso, é um exemplo desse processo.

A partir deste processo, grande parte dos problemas sociais, ambientais e até econômicos de uma cidade é creditado ao chamado crescimento desordenado. Assim, Braga (2016) afirma que o mesmo modo que as baixas densidades, as altas densidades podem acarretar diversos problemas urbanos, inclusive deseconomias de aglomeração.

Entretanto, as cidades que se reinventam devem se atentar à inclusão social e tecnologias verdes, aliadas à gestão inteligente do território para o desenvolvimento urbano sustentável de novos territórios.

Os princípios da sustentabilidade devem ser inseridos no planejamento. Observa-se o conceito de cidade sustentável dado por Ferrão (2016) como o conjunto urbano formado por espaços de vivência comunitária com base em projetos de cidade saudável e solidária, planejada para minimizar seus impactos sobre o ambiente.

Para que haja reflexões do modelo de desenvolvimento urbano, otimizando o uso da infraestrutura urbana e promovendo a maior sustentabilidade, novos modelos de funcionamento, gestão e crescimento estão na análise da expansão urbana no interior da América do Sul.

\section{A URBANIZAÇÃO NO INTERIOR DA AMÉRICA DO SUL}

Percebe-se a partir da segunda metade do século XX, segundo Naslavsky (2016), a concretização de obras modernistas nas regiões Norte, Sul, Nordeste e Centro-Oeste. Essas manifestações da arquitetura moderna brasileira foram difundidas em Curitiba-PR, com o Teatro Guaíra, por exemplo (SANTOS, 2006). Assim como também chegaram em terras matogrossenses.

O autor Vilarinho Neto (2009) descreve a criação do município de Várzea Grande em 23 de setembro de 1948, dentro do período de difusão do modernismo brasileiro. Silva (2016) aponta um crescimento mais efetivo em Várzea Grande-MT somente no século XX, a partir de 1942, quando o interventor Júlio Müller inaugurou a ponte de concreto unindo Várzea Grande a Cuiabá, possibilitando a chegada da energia elétrica em 1945.

De acordo Silva (2016), a emancipação de Várzea Grande alterou a dinâmica urbana e espacial da cidade com conformação de uma área central, assim como contribuiu para a expansão urbana.

Acerca da modernização, Colosso (2016) afirma que Lefebvre considera a modernização engendrada por um duplo processo: forças técnico-produtivas (a industrialização) e a urbanização da sociedade. Esse processo é identificado a partir dos estudos urbanos, que apontados por Silva (2016), são imóveis localizados próximos da área central da cidade, possibilitando uma elevação do valor dos mesmos e os empreendimentos, visando lucro, corporativizam o espaço da cidade.

Seguindo esta lógica, é recorrente em áreas centrais da cidade ocorrer a expulsão de moradores para as áreas mais afastadas da área central, negando o direito do cidadão à cidade (SILVA, 2016, p.29). Colosso (2016), novamente, baseado nos estudos de Lefebvre, informa que esse processo de modernização foi acompanhado por uma divisão social e 
técnica do trabalho, das práticas e dos saberes. Dessa maneira, a realidade social não dimensiona a urbanização moderna (ver Figura 1).

Figura 1 - Igreja de Nossa Senhora do Carmo, ladeada pelas Avenidas Filinto Muller e Couto Magalhães.

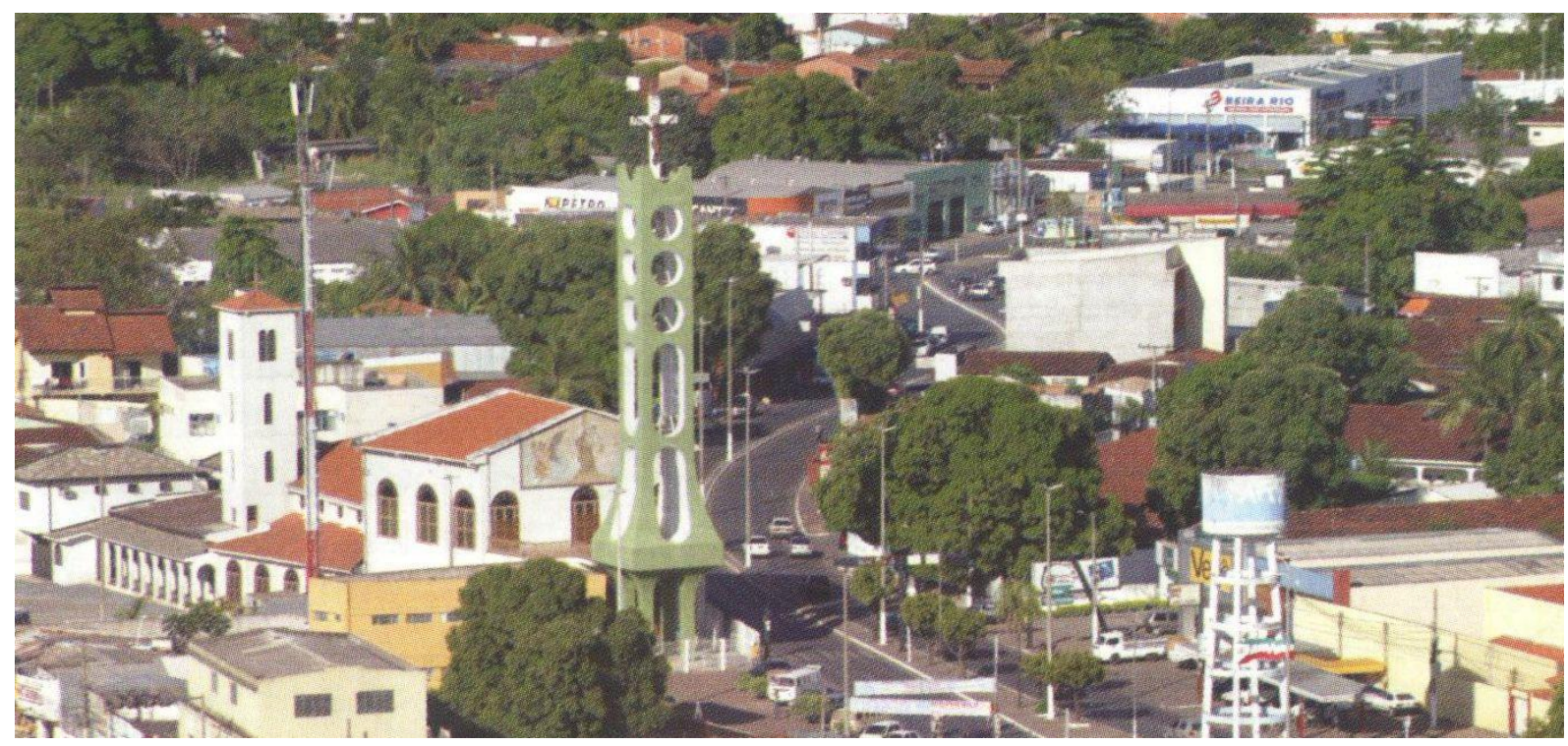

Fonte: Blog Cultural Várzea Grande-MT (Antiga): <http://varzeaantiga.blogspot.com/201 1/07/centrode-varzea-grande.html>. Acesso em 09 Jun 2019.

Fazendo oposição aos estudos de Lefebvre, através das premissas e indicadores de avaliação relacionados, as cidades inteligentes e sustentáveis se apresentam como uma possibilidade para a mitigação dos principais problemas que vêm afetando as cidades, como por exemplo, a polvição, o gasto energético em demasia, os congestionamentos e o aquecimento (BACHENDORF et al, 2018, p. 207).

\section{USOS DE SOLO}

Apesar de Várzea Grande ser separada de Cuiabá apenas pelo Rio Cuiabá, as cidades devem ser analisadas a partir de diversos pontos de vista. Por exemplo, sob o ponto de vista climático, as atividades humanas que emitem gases de efeito estufa o fazem para atender às demandas desta população, estejam estas atividades sendo realizadas dentro ou fora das cidades (KLUG et al., 2016).

A partir da observação dos tipos de infraestrutura da cidade, citados por Maropo et al. (2019), estes podem ser classificados em cinza ou verde. O autor explica que a infraestrutura convencional, a cinza, é representada pelas ruas, sistemas de rede esgoto e água canalizados podem ser substituídos pela infraestrutura verde, podendo recuperar áreas degradadas em virtude de uma urbanização desenfreada. Pensando economicamente, o uso das tecnologias verdes representa processos mais econômicos que ao uso da infraestrutura convencional.

Esclarece-se que, segundo Spinosa et al. (2018), o planejamento é um instrumento importante para a gestão urbana e no Brasil, por força de lei, possui o Estatuto da Cidade, que estabelece diretrizes para o desenvolvimento urbano em várias dimensões. Além desse planejamento legal, o planejamento deve ser inteligente, como apontam Ahvenniemi et al. (2017), ao citar que as cidades estão se esforçando no sentido de tornarem-se mais inteligentes e por consequência os objetivos de cidades inteligentes estão estreitamente interligados aos objetivos das cidades sustentáveis.

Contudo, Oliveira \& Carvalho (2019) explicam que o modelo segregador está presente nos espaços, com conjuntos de escritórios, shoppings centers, escolas, centros de lazer, parques temáticos e condomínios horizontais fechados. Esses espaços são definidos como enclaves 
fortificados. Destaca-se a observação de Silva (2016), ao citar o Várzea Grande Shopping como um dos maiores cenários de inserção no processo de evolução da cidade. Assim, é possível verificar a relação da evolução da cidade e da segregação.

A partir do conceito de enclave fortificado, dado por Oliveira \& Carvalho (2019), o novo campus da Universidade Federal de Mato Grosso (UFMT), o campus do Instituto Federal de Mato Grosso (IFMT) e o campus da Universidade Estadual de Mato Grosso (Unemat) são responsáveis pelo processo de grande transformação do espaço (Ver Figura 2).

Figura 2 - Mapa da expansão urbana de Várzea Grande.

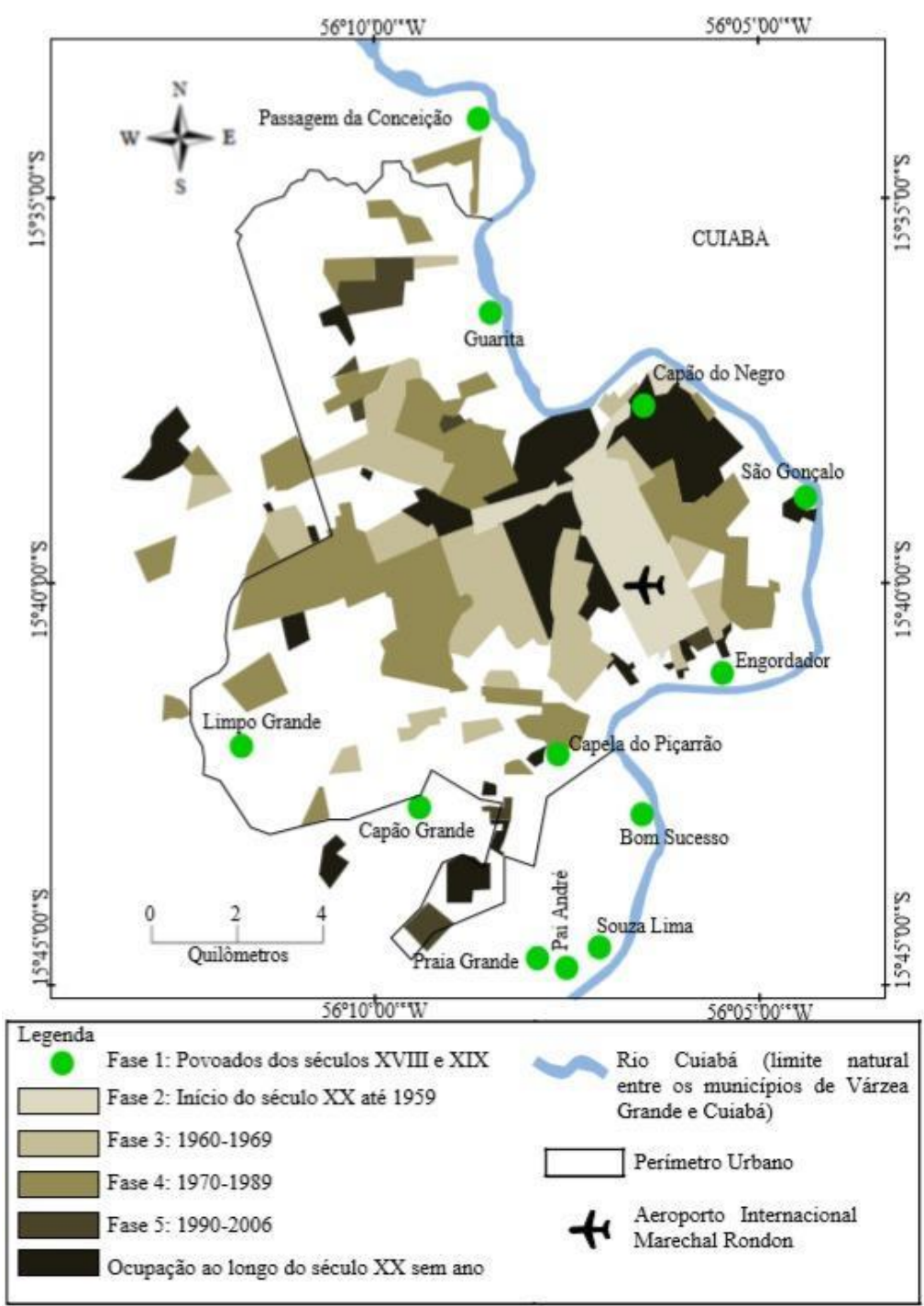

Fonte: Oliveira (2016)

Como consequência desse planejamento urbano, descrito por Silva (2016), essa região está em expansão. A inserção de enclaves fortificados, além de aumentar a velocidade dos investimentos, produzem novos rearranjos socioterritoriais.

Gusmão \& Bovo (2019) afirmam que a presença de espaços ociosos na área urbana é uma realidade que atinge as cidades de diferentes tamanhos. Em contraponto aos vazios urbanos, Amorim (2019) destaca que a associação da alta densidade de construções com a menor presença de cobertura vegetal arbórea são os principais fatores responsáveis pelas maiores intensidades nas temperaturas dos alvos.

Silva (2016) cita que o processo de urbanização, diante das necessidades da reprodução do capital, é o espaço produzido socialmente e tomado como mercadoria. As reflexões de 
Oliveira \& Carvalho (2019) indicam reprodução maciça das paisagens urbanas nas áreas de expansão de Várzea Grande, como decorrência do próprio desenvolvimento da economia e, por consequência, exigindo e construindo um modo de superar essa reprodução.

Diferentemente das paisagens homogêneas de condomínios horizontais, a Copa do Mundo de 2014, um megaevento, produziu vários enclaves fortificados. Pontua-se que Várzea Grande, apesar de não ter sido escolhida a cidade-sede para a Copa do Mundo 2014, recebeu a influência dos investimentos realizados para o evento na cidade vizinha, Cuiabá (ver Figura 3).

Figura 3 - Implantação do VLT na Avenida da FEB em Várzea Grande.

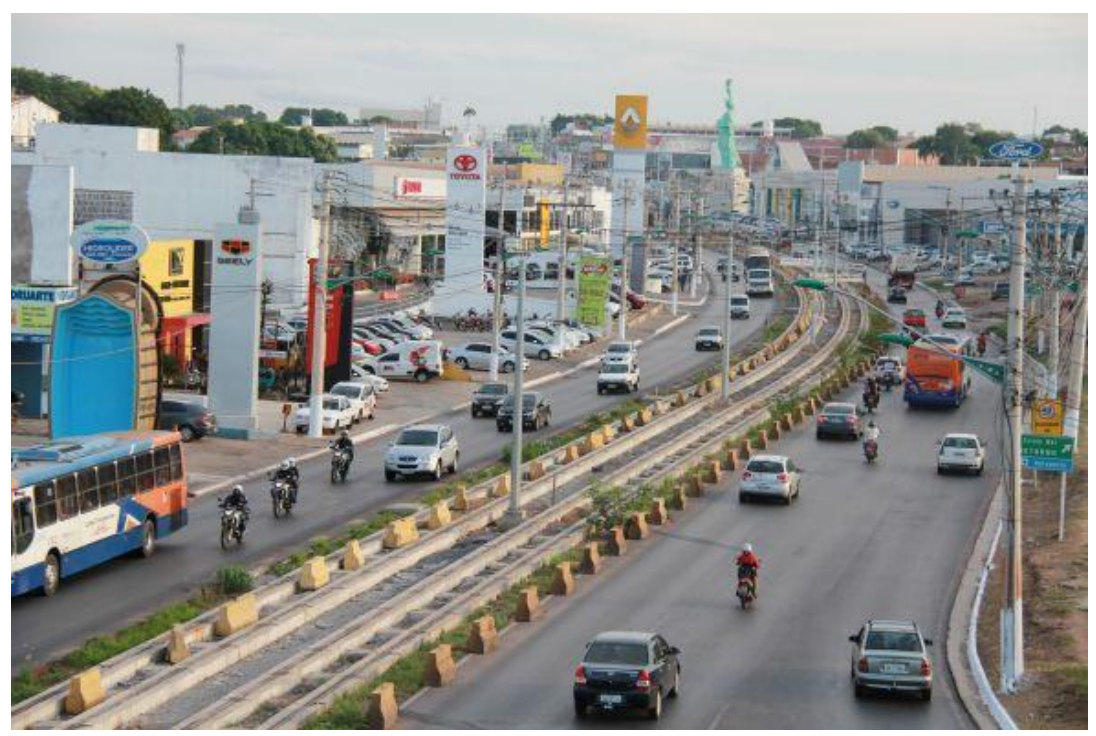

Fonte: Gazeta Digital. Disponível em

<http://www.gazetadigital.com.br/editorias/economia/avenida-da-feb-enterraempresas/479691>. Acesso em 09 Jun 2019.

No processo de expansão urbana, Silva (2016) pontua que o planejamento deve tomar cuidado no conflito direto com os habitantes, que muitas vezes se sentem expulsos de seus bairros e de suas casas em função do processo causado pela renovação urbana. Assim, urbanização de Várzea Grande caracteriza-se pela policentralidade, baseada em Ferreira (2018).

\section{CONCLUSÕES}

A respeito do desenvolvimento urbano sustentável, sabe-se que é um processo de transformação econômico, social e ambiental. Os recursos e investimentos podem ser explorados promovendo o desenvolvimento tecnológico. Isso gera o equilíbrio entre todos os setores envolvidos.

Dessa forma, a equidade urbana é alcançada de forma inteligente através da integração sistêmica de todos elementos que levam a um ordenamento territorial baseado na conexão patrimônio-paisagem cultural, fazendo a população local se sentir parte da paisagem urbana, a partir de empreendimentos ecologicamente corretos e economicamente adequados para a conformação de cidades sustentáveis, atendendo também a configuração de cidades inteligentes.

Logo, o planejamento urbano exige atenção para que os obstáculos físicos e os distanciamentos não simbolizem o desejo de afastamento e a afirmação de diferenciação social. Portanto, a segregação socioterritorial não faz parte de uma cidade sustentável.

Todavia, apesar da segregação nas cidades atuais, a população deve estar inserida dentro de um processo urbano dinâmico na qual ela é o usuário final de um produto, a cidade. 0 
futuro deste produto está ligado a questões urbanas sustentáveis e inteligentes. Assim, o papel das cidades é o principal motor das transformações desejadas.

\section{AGRADECIMENTOS}

Agradeço ao UNIVAG - Centro Universitário de Várzea Grande pelo apoio dado no desenvolvimento do trabalho relatado neste artigo.

\section{REFERÊNCIAS}

AHVENNIEMI, H.; HUOVILA, A.; PINTO-SEPPÄ, I.; AIRAKSINEN, M. What are the differences between sustainable and smart cities? Cities, v. 60, p. 234-245, 2017.

AMORIM, M. C. de C. T.. Ilhas de calor superficiais: frequência da intensidade e variabilidade espacial em cidade de clima tropical continental. Geo UERJ, Rio de Janeiro, n. 34, e,40959, 2019. DOI:10.12957/geoverj.2019.40959.

BACHENDORF, C. F.; SANTOS, G. D.; PEZARICO, G.; SILVA, M. P.. Municípios paranaenses sob a ótica dos indicadores de cidades inteligentes e sustentáveis: uma análise estatística. BARU, Revista de Assuntos Regionais e Urbanos. Goiânia, GO, v. 4, n. 2, p. 206-222, jul./dez. 2018. eISSN 2448-0460.

BRAGA, R. Avaliação da sustentabilidade da expansão do perímetro urbano da cidade de Piracicaba - SP por meio de indicadores de forma urbana. Geografia, Ensino \& Pesquisa, v. 20, n.2, p. 33-44, 2016. ISSN: 2236-4994. DOI: 10.5902/2236499420699.

COLOSSO, P. A Crítica de Henri Lefebvre ao Urbanismo Moderno. In: SEMINÁRIO DE HISTÓRIA DA CIDADE E DO URBANISMO, 14., 2016, São Carlos. Anais... São Carlos: USP, 2016. p. 80-88.

FERRÃO, A. M. de A. Cidades e territórios sustentáveis, paisagens e desenvolvimento regional. Labor \& Engenho, Campinas, SP, v. 10, n. 2, p. 170-179, abr./jun. 2016. DOI: http://dx.doi.org/10.20396/lobore.v10i2.8646238.

FERREIRA, H. M. A centralidade intraurbana a partir da análise de franquias e filiais de cidades medias. Revista RA'EGA - O Espaço Geográfico em Análise, Curitiba, PR, v. 45, n. 1, p. 123-141, dez. 2018. DOI: 10.5380/raega.

GUSMÃO, P. S.; BOVO, M. C. Análise dos Impactos dos Vazios Urbanos na Produção do Espaço da Cidade de Terra Boa (PR), Brasil. Geografia (Londrina) v. 28. n. 1. pp. 81 - 96, Fev. 2019. ISSN 2447-1747. DOI: 10.5433/2447-1747.2019v28n 1 p81.

KLUG, L.; MARENGO, J. A.; LUEDEMANN, G. Mudanças climáticas e os desafios brasileiros para implementação da nova agenda urbana. 2016. In book: O Estatuto da Cidade e a Habitat III : um balanço de quinze anos da política urbana no Brasil e a nova agenda urbana, Chapter: 12, Publisher: IPEA, Editors: Marco Aurélio Costa, pp.303 - 322. Disponível em: <https://www.researchgate.net/publication/308688985 >. Acesso em 18 abr. 2019.

MAROPO, V. L. B; MORAIS, E. E; NUNES, A. C; SILVEIRA, J. A. R. Planejamento urbano sustentável: um estudo para implantação de infraestrutura verde no Bairro Bancários, João Pessoa-PB, Brasil. Urbe, Revista Brasileira de Gestão Urbana, 11, e20180005. João Pes DOI: https://doi.org/10.1590/2175-3369.011.002.AO09.

NASLAVSKY, G.; LARA, F. Regionalismo como alteridade: da América Latina ao Nordeste do Brasil, reflexões sobre a historiografia da arquitetura moderna brasileira. In: $11^{\circ}$.SEMINÁRIO DO DOCOMOMO BRASIL. Anais... Recife: DOCOMOMO_BR, 2016. Pp.1-9. 
OLIVEIRA, A. F. de; CARVALHO, S. L. de S. Condomínios horizontais fechados, periferia e segregação socioterritorial urbana. Resenha sobre Palmas: uma cidade sustentável. Palmas: EDUFT, 2019. ISBN: 978-85-60487-55-4

OLIVEIRA, J. H. D. de. Transformações socioespaciais em Várzea Grande-MT: Entre negócios urbanos e remoções de famílias no contexto da realização da Copa do Mundo de 2014. Universidade Federal de Mato Grosso. Dissertação de Mestrado. Cuiabá: 2016.

RIBEIRO, C. R.; GONÇALVES, A. P.; BASTOS, F. P. llhas de calor urbanas e conforto térmico humano em cidades de porte médio: estudo aplicado em Juiz de Fora (MG). Revista Ra'ega - O espaço geográfico em análise. Curitiba, v. 45, n. 1, p. 281-300, Dezembro 2018. elSSN: 2177-2738. DOI: 10.5380/raega.

SANTOS, M. da G. R.. Arquitetura moderna brasileira: dos pioneiros a Brasília. Revista Da Vinci, Curitiba, v. 3, n. 1, p. 37-56, 2006

SILVA, A. V. da. Formação do espaço urbano e as formas de habitação na cidade de Várzea Grande MT. UFMT. Dissertação de Mestrado. Cuiabá: 2016.

SPINOSA, L. M.; KRAMA, M.; HARDT, C. Desenvolvimento urbano baseado em conhecimento e ecossistemas de inovação urbanos: uma análise em quatro cidades brasileira. Revista Latino Americana de Estudos Urbanos Regionais (EURE). Santiago de Chile, volume 44, n. 131, p. 193-214, jan. 2018.

VILARINHO NETO, C. S. A metropolização regional, formação e consolidação da rede urbana do estado de Mato Grosso. Cuiabá: EdUFMT, p. 140, 2009. 